\title{
Random Delay Differential Equations and Inverse Problems for Aggregate Data Problems
}

\author{
H. T. Banks ${ }^{1}$, and W. Clayton Thompson ${ }^{1,2}$ \\ July 28, 2018 \\ ${ }^{1}$ Center for Research in Scientific Computation \\ Department of Mathematics \\ North Carolina State University \\ Raleigh, NC \\ and \\ ${ }^{2}$ SAS Institute \\ Cary, NC
}

\begin{abstract}
We consider nonparametric estimation of probability measures for parameters in delay differential equation (DDE) problems where only aggregate (population level) data are available. We summarize an existing computational method for the estimation problem which has been developed over the past several decades $[11,17,21,26,28]$. Theoretical results are presented which establish the existence and consistency of very general (ordinary, generalized and other) least squares estimates and estimators for the measure estimation problem with specific application to random DDEs.
\end{abstract}

Key Words: Inverse problems, random delay differential equations, aggregate data, approximation and consistency of estimators.

AMS Classifications: 62G07, 34A55, 46S50, 93E24. 


\section{Introduction}

We extend the theory of aggregate data inverse problems of [25] to general nonlinear non-autonomous delay differential equations (DDEs) of the form

$$
\begin{aligned}
\dot{x}(t) & =G\left(t, x(t), x_{t}, x\left(t-\tau_{1}\right), \ldots, x\left(t-\tau_{m}\right), \theta\right)+G_{2}(t), \quad 0 \leq t \leq T, \\
x_{0} & =\phi,
\end{aligned}
$$

where $G=G\left(t, \eta, \psi, y_{1}, \ldots, y_{m}\right):[0, T] \times X \times \mathbb{R}^{n m} \times \mathbb{R}^{p} \rightarrow \mathbb{R}^{n}$. Here $X=$ $\mathbb{R}^{n} \times L_{2}\left(-r, 0 ; \mathbb{R}^{n}\right), 0<\tau_{1}<\ldots<\tau_{m}=r, x_{t}$ denotes the usual function $x_{t}(\xi)=x(t+\xi),-r \leq \xi \leq 0$, while $x(t)$ is a point in $\mathbb{R}^{n}$, and $\phi \in H^{1}(-r, 0)$. The function $G_{2}$ is a time dependent perturbation (e.g., a control input).

This involves extending the existing theory for random ODEs of [25] to allow treatment of general nonlinear random delay differential equations (RDDEs) in the context of aggregate data inverse problems using the Prohorov Metric Framework as described in [4]. This allows treatment of inverse problems when individual longitudinal data is not available. We refer to this class of problems as Type II problems wherein one has only aggregate or population level longitudinal data available. This is common in marine, insect, etc., catch and release experiments [22] where one samples at different times from the same population but cannot be guaranteed of observing the same subset of individuals at each sample time under constant environmental, etc., conditions. This type of data is also typical in biomedical experiments where the organism or population member being studied is sacrificed in the process of making a single observation (e.g., certain physiologically based pharmacokinetics (PBPK) modeling $[1,23,30,31,32]$ and many other biomedical problems. In this case one may still have dynamic (i.e., time course) models for individuals, but no individual data are available. We are motivated in this presentation by the cellular problems for HIV infection as studied in $[6,10,11]$ using delay differential equations.

Our ideas of using distributions for parameters in inverse problems grew out of work in [12] and use of Sinko-Streifer models for mosquitofish growth rates where only aggregate data were available due to periodic sampling of different subsets of the population. The first theoretical results in the context of inverse problems were in [17]. There were numerous subsequent uses including shrimp population size models in $[9,16]$ and carboxyfluorescein succinimidyl ester (CFSE) labeling models [18].

To briefly describe our class of problems, we consider approximation methods in estimation or inverse problems but the quantity of interest is a probability

distribution. Assume we have a parameterized system $(q \in \Omega)$ with state model responses $x(t ; q)$ describing the population of interest. For data or observations, we are given a set of values $\left\{y_{l} \approx C x\left(t_{l} ; q\right)\right\}$ for the expected values

$$
\mathcal{E}\left[y_{l}(q) \mid P\right]=\int_{\Omega} y_{l}(q) d P(q)
$$

with respect to the unknown probability distribution $P$ describing the distribution of parameters $q$ over the population. We use data to choose from a 
given family of distributions $\mathcal{P}(Q)$ the distribution $P^{*}$ that gives the best fit of the underlying model to data. This is accomplished by formulating an ordinary least squares (OLS) problem; however we note that we could equally well use a weighted least squares (WLS) or maximum likelihood estimation (MLE) framework (e.g., see [19] for relationships between MLE and OLS estimators). Specifically we seek to minimize

$$
J(P)=\sum_{l}\left|\mathcal{E}\left[y_{l}(q) \mid P\right]-y_{l}\right|^{2}
$$

over $P \in \mathcal{P}(\Omega)$. Even for simple dynamics for $y_{l}(q)$ this yields an infinite dimensional optimization problem. Therefore one needs approximations that lead to computationally tractable schemes. That is, it is useful to formulate methods to yield finite dimensional sets $\mathcal{P}^{M}(\Omega)$ over which to minimize $J(P)$. Of course, we wish to choose these methods so that " $\mathcal{P}^{M}(\Omega) \rightarrow \mathcal{P}(\Omega)$ " in some sense. In our case we shall use the Prohorov Metric $[11,26]$ of weak star convergence of measures to assure the desired approximation convergences as well as consistency of the estimators.

\section{Prohorov Estimates and Their Approximations}

Convergence in the Prohorov metric is equivalent to the weak* convergence of measures when the space of probability measures $\mathcal{P}(\Omega)$ is imbedded in the dual $C^{*}(\Omega)$ of the space of bounded continuous functions on $\Omega$. We discuss briefly existence, convergence, and consistency theory. In the discussions we adopt the following notation : $N=N_{t}$ are the number of data points or observations; $v^{\tilde{N}}(t ; \omega)$ are the state approximations to $v(t ; \omega)$, so $\tilde{N}$ is the index for state approximations; $M$ will be the parameter approximation index

We assume a family $\mathcal{P}(\Omega)$ of permissible probability functions for our parameters. We attempt to perform the estimation in a least-squares framework

$$
\hat{P}_{N}=\underset{P \in \mathcal{P}(\Omega)}{\operatorname{argmin}} J_{N}(v, P)=\underset{P \in \mathcal{P}(\Omega)}{\operatorname{argmin}} \sum_{j=1}^{N}\left(v_{j}-v\left(t_{j} ; P\right)\right)^{2}
$$

where $v\left(t_{j} ; P\right)$ is given in $(7)$ and $N$ observations are used to obtain a best fit for a nominal or "true" parameter $P_{0}$. In order to approximate this minimizer, we replace the infinite dimensional optimization problem by a sequence of finitedimensional optimization problems with, for example, Dirac or spline-based distributions. Thus, if we use the Dirac approximating families, we set $\boldsymbol{Q}^{M}=$ $\left\{\Delta_{q_{k}}, k=1, . ., M\right\}$, where $M$ represents the number of nodes, or elements, used in the approximation. Our family of approximating probability functions becomes

$$
\mathcal{P}^{M}(\Omega)=\left\{P^{M}=\sum_{k=1}^{M} w_{k} \Delta_{q_{k}} \mid w_{k} \geq 0 \text { and } \sum_{k=1}^{M} w_{k}=1\right\},
$$


where $\Delta_{q_{k}}$ represents the Dirac delta function at the point $q_{k}$ and $w_{k}$ are the weights and/or probabilities. It has been previously proven $[4,28]$ that there exists a minimizer for the discrete approximation problem

$$
\hat{P}_{N}^{M}=\underset{P \in \mathcal{P}^{M}(\Omega)}{\operatorname{argmin}} \sum_{j=1}^{N_{t}}\left(v_{j}-v\left(t_{j} ; P\right)\right)^{2} .
$$

A further approximation arises when we appproximate the state variable $v$ by numerical approximation $v^{\tilde{N}}$, e.g., by finite elements for example, and seek to solve

$$
\hat{P}_{N, M}^{\tilde{N}}=\underset{P \in \mathcal{P}^{M}(\Omega)}{\operatorname{argmin}} \sum_{j=1}^{N}\left(v_{j}-v^{\tilde{N}}\left(t_{j} ; P\right)\right)^{2} .
$$

There are a number of questions that arise immediately in the class of problems we have defined. Perhaps the most obvious are questions of convergence (what happens as $M \rightarrow \infty$ in the Dirac or spline approximations?) and consistency (what happens as $N=N_{t} \rightarrow \infty$ ?) These questions have been successfully investigated both theoretically $([4,25]$ and the references therein) and computationally $([14,15]$ and the references therein) for certain classes of problems. A further issue involves the delay differential equation approximations $v^{\tilde{N}}$ (e.g., finite element approximations of the realizations of the the random DDE (6) to the solution). Again, elements of the necessary convergence issues have been addressed in $[22, ?]$ in certain cases and here we wish to extend these ideas to the case of delay differential equations systems. In summary we wish to establish for the problems discussed here that the approximations $\hat{P}_{N, M}^{\tilde{N}}$ converge to a "true" distribution $P_{0}$ as the number of elements used in the approximations increases (i.e., $M, N, \tilde{N} \rightarrow \infty$ ).

Significantly, the Prohorov Metric Framework is computationally constructive. That is, in practice, one does not construct a sequence of estimates for increasing values of $M$ and $\tilde{N}$; rather, one fixes the values of $M$ and $\tilde{N}$ to be sufficiently large to attain a desired level of accuracy. To do this we need only to have some enumeration of the elements of $\mathcal{P}^{M}(\Omega)$ in order to compute an approximate estimate $\hat{P}_{N, M}^{\tilde{N}}$. This leads to a constrained quadratic optimization problem [25].

The novel results in [25] establishes the desirable property of consistency of the estimator $P_{N}$ as a measurable function mapping the data observation process to the space of probability measures. However, it is generally not possible to directly solve the optimization problems (2) for $\hat{P}_{N}$ as a function of $v$. As a result, approximate (generally numerical) methods must be used in order to solve (4) and obtain an approximate estimate $\hat{P}_{N, M}^{\tilde{N}}$. We must ascertain, then, how the approximate estimate $\hat{P}_{N, M}^{\tilde{N}}$ relates to the exact estimate $\hat{P}_{N}$ (for any fixed value of $N$.) 


\section{Problem formulation}

To simplify our discussions we take $q=\left(\tau_{1}, \theta\right)$, i.e., we consider only one delay $\tau_{1}$ and a general system parameter $\theta \in R^{p}$ for a one dimensional vector system (arguments for the $n$ dimensional system with multiple delays readily follow using the same arguments!) of the form

$$
\begin{aligned}
\dot{x}(t) & =G\left(t, x(t), x_{t}, x\left(t-\tau_{1}\right), \theta\right)+G_{2}(t), \quad 0 \leq t \leq T, \\
x_{0} & =\phi,
\end{aligned}
$$

which, in the form of random delay differential equations, is given by

$$
\begin{aligned}
\dot{x}(t) & =G\left(t, x(t), x_{t}, \int_{\Omega_{1}} x\left(t-\tau_{1}\right) d P_{1}\left(\tau_{1}\right), \int_{\Omega_{2}} \theta d P_{2}(\theta)\right)+G_{2}(t), \quad 0 \leq t \leq T, \\
x_{0} & =\phi,
\end{aligned}
$$

where $P_{1}: \Omega_{1} \subset R_{1}^{+} \rightarrow[0,1], P_{2}: \Omega_{2} \subset R^{p} \rightarrow[0,1]^{p}$ are probability measures on compact sets $\Omega_{1}$ and $\Omega_{2}$ in $R_{1}^{+}$and $R^{p}$, respectively.

We are interested in inverse problems for a given data set $v=\left\{v_{j}\right\}$ for the expected value

$$
v\left(t_{j}\right)=\mathbb{E}\left[x\left(t_{j} ; \tau_{1}, \theta\right) \mid P_{1}, P_{2}\right]=\int_{\Omega_{1} \times \Omega_{2}} x\left(t_{j} ; \tau_{1}, \theta\right) d P_{1}\left(\tau_{1}\right) d P_{2}(\theta) .
$$

The corresponding cost functionals are defined by

$$
\left.J_{N}\left(v, P_{1}, P_{2}\right)\right)=\sum_{j=1}^{N}\left(v_{j}-\int_{\Omega_{1} \times \Omega_{2}} x\left(t_{j} ; \tau_{1}, \theta\right) d P_{1}\left(\tau_{1}\right) d P_{2}(\theta)\right)^{2} .
$$

\subsection{Solutions and Approximations}

We first summarize existence and uniqueness for general nonlinear non-autonomous dynamical systems involving delays of the form

$$
\begin{aligned}
\dot{x}(t) & =G\left(t, x(t), x_{t}, x\left(t-\tau_{1}\right), \theta\right)+G_{2}(t), \quad 0 \leq t \leq T, \\
x_{0} & =\phi,
\end{aligned}
$$

where $G=G\left(t, \eta, \psi, y_{1}, \theta\right):[0, T] \times X \times \mathbb{R}^{1} \times \mathbb{R}^{p} \rightarrow \mathbb{R}^{1}$. Here $X=\mathbb{R}^{1} \times$ $L_{2}\left(-r, 0 ; \mathbb{R}^{1}\right), 0<\tau_{1} \leq r, x_{t}$ denotes the usual function $x_{t}(\xi)=x(t+\xi)$, $-r \leq \xi \leq 0$, while $x(t)$ is a point in $\mathbb{R}^{1}$, and $\phi \in H^{1}(-r, 0)$. The function $G_{2}$ is a time dependent perturbation (e.g., a possible control input).

We turn to the mathematical aspects of these nonlinear functional differential equation (FDE) systems and present an outline of the necessary mathematical foundations. First we describe the conversion of the nonlinear FDE system to an abstract evolution equation (AEE) as well as provide existence and uniqueness 
results for a solution to the FDE. One can use the ideas of a linear semigroup framework, in which approximation of linear delay systems has been developed, as a basis for a wide class of nonlinear delay system approximations. Details in this direction can be found in the early work $[2,3,29]$ which are direct extensions to nonlinear delay systems of the linear system results in $[7,8,20]$. We shall make use of the following hypotheses throughout our presentation.

(H1) The function $G$ satisfies a global Lipschitz condition:

$$
\begin{aligned}
&\left|G\left(t, \eta, \psi, y_{1}, \theta\right)-G\left(t, \tilde{\eta}, \tilde{\psi}, w_{1}, \tilde{\theta}\right)\right| \\
& \leq K\left(|\eta-\tilde{\eta}|+|\psi-\tilde{\psi}|+\left|y_{1}-w_{1}\right|+|\theta-\tilde{\theta}|\right)
\end{aligned}
$$

for some fixed constant $K$ and all $\left(\eta, \psi, y_{1}, \theta\right),\left(\tilde{\eta}, \tilde{\psi}, w_{1}, \tilde{\theta}\right)$ in $X \times \mathbb{R}^{1} \times \mathbb{R}^{p}$ uniformly in $t$.

(H2) The function $G:[0, T] \times X \times \mathbb{R}^{1} \times \mathbb{R}^{p} \rightarrow \mathbb{R}^{1}$ is differentiable in each argument with all derivatives (ordinary and partial) dominated by integrable functions.

Letting $z(t)=\left(x(t), x_{t}\right) \in X$, where the Hilbert space $X$ has the inner product

$$
\langle(\eta, \phi),(\zeta, \psi)\rangle_{X}=<\eta, \zeta>_{\mathbb{R}^{1}}+\int_{-r}^{0} \phi(\xi) \psi(\xi) d \xi,
$$

we define the nonlinear operator $\mathcal{A}(t): \mathcal{D}(\mathcal{A}) \subset X \rightarrow X$ by

$$
\begin{gathered}
\mathcal{D}(\mathcal{A}) \equiv\left\{(\psi(0), \psi) \mid \psi \in H^{1}(-r, 0)\right\} \\
\mathcal{A}(t)(\psi(0), \psi)=\left(G\left(t, \psi(0), \psi, \psi\left(-\tau_{1}\right), \theta\right), D \psi\right)
\end{gathered}
$$

where here $D \psi=\psi^{\prime}$. Then the FDE (9) can be formulated as

$$
\begin{aligned}
\dot{z}(t) & =\mathcal{A}(t) z(t)+G_{2}(t) \\
z(0) & =z_{0},
\end{aligned}
$$

where $z_{0}=\left(x_{0}, \phi\right)$ is the initial condition.

Theorem 3.1. Assume that (H1) holds and let $z\left(t ; \phi, G_{2}\right)=\left(x\left(t ; \phi, G_{2}\right), x_{t}\left(\phi, G_{2}\right)\right)$, where $x$ is the solution of (9) corresponding to $\phi \in H^{1}, G_{2} \in L_{2}$. Then for $\zeta=(\phi(0), \phi), z\left(t ; \phi, G_{2}\right)$ is the unique solution on $[0, T]$ of

$$
z(t)=\zeta+\int_{0}^{t}\left[\mathcal{A}(\sigma) z(\sigma)+\left(G_{2}(\sigma), 0\right)\right] d \sigma .
$$

Furthermore, the mapping $G_{2} \rightarrow z\left(t ; \phi, G_{2}\right)$ is weakly sequentially continuous from $L_{2}$ (with weak topology) to $X$ (with strong topology). 
These results can be established in one of several ways [3] including fixed point theorem arguments or Picard iteration arguments; either approach can be used to establish existence, uniqueness and continuous dependence of the solution of (12). More complete discussions can be found in [4] and the detailed references given therein.

We let $Z^{\tilde{N}}$ be the approximating linear spline subspaces (see $[2,3,4,20]$ ) of $Z$ such that

$Z^{\tilde{N}}=$

$\left\{(\phi(0), \phi) \mid \phi\right.$ a continuous, linear spline with nodes at $\left.\left\{t_{j}^{\tilde{N}}\right\}=\{-j r / \tilde{N}\}_{j=0}^{\tilde{N}}\right\}$,

and $P^{\tilde{N}}$ be the orthogonal projection in $\langle\cdot, \cdot\rangle_{X}$ of $Z$ onto $Z^{\tilde{N}}$. For the approximating operator $\mathcal{A}^{\tilde{N}}(t)=P^{\tilde{N}} \mathcal{A}(t) P^{\tilde{N}}$, we define the approximating solutions in $Z^{\tilde{N}}$

$$
z^{\tilde{N}}(t)=P^{\tilde{N}} \zeta+\int_{0}^{t}\left[A^{\tilde{N}}(\sigma) z^{\tilde{N}}(\sigma)+P^{\tilde{N}}\left(G_{2}(\sigma), 0\right)\right] d \sigma,
$$

which are the unique solutions of the differential equations

$$
\begin{aligned}
\frac{d z^{\tilde{N}}}{d t} & =\mathcal{A}^{\tilde{N}}(t) z^{\tilde{N}}(t)+P^{\tilde{N}}\left(G_{2}(t), 0\right), \\
z^{\tilde{N}}(0) & =P^{\tilde{N}} \zeta .
\end{aligned}
$$

We note that these equations are finite dimensional ordinary differential equations and hence existence of solutions are readily guaranteed.

Theorem 3.2. Assume (H1), (H2). Let $\zeta=(\phi(0), \phi), \phi \in H^{1}$ and $G_{2} \in$ $H^{0}(0, T)$ be given, with $z^{\tilde{N}}$ and $z$ the corresponding solutions on $[0, T]$ of (14) and (11), respectively. Then $z^{\tilde{N}}(t) \rightarrow z(t)=\left(x\left(t ; \phi, G_{2}\right), x_{t}\left(\phi, G_{2}\right)\right)$ as $\tilde{N} \rightarrow \infty$, uniformly in $t$ on $[0, T]$.

The proof of this theorem follows from arguments in [5]. Indeed it is given as Theorem 2.3 in [5]. In fact the uniform convergence can be extended to parameters $q=\left(\tau_{1}, \theta\right)$ lying in compact sets $\Omega_{1} \times \Omega_{p}$ by consideration of the arguments in [5]. Indeed a careful reading of the proofs in [5] reveals that the dissipative inequality (7) of [5] holds uniformly for $q=\left(\tau_{1}, \theta\right)$ in compact sets $\Omega=\Omega_{1} \times \Omega_{p}$. This is also true for the corresponding approximate dissipative inequality (11) in [5]. This leads to the statement of the convergence in Theorem 3.2 holding uniformly in $t$ on $[0, T]$ and in $q=\left(\tau_{1}, \theta\right)$ on compact sets $\Omega_{1} \times \Omega_{p}$. In related considerations, one can use the arguments behind Lemma 3 of [24] and the arguments discussed there to argue that solutions are continuous with respect to delays and parameters uniformly on compact restraint sets $\Omega=\Omega_{1} \times$ $\Omega_{p}$. That is, $x\left(t ; \tau_{1}, \theta\right)$ is continuous on compact sets $\Omega_{1} \times \Omega_{p}$. This allows us to argue the following preliminary results which we will need. 
Theorem 3.3. Suppose $P_{k} \rightarrow P$ in $\mathcal{P}(\Omega)=\mathcal{P}\left(\Omega_{1} \times \Omega_{p}\right)$. Then for each $t$ as $\tilde{N}, k \rightarrow \infty$ we have

$$
v^{\tilde{N}}\left(t ; P_{k}\right)=\int_{\Omega} x^{\tilde{N}}(t ; \omega) d P_{k}(\omega) \rightarrow v(t ; P)=\int_{\Omega} x(t ; \omega) d P(\omega) .
$$

Proof.

For each fixed $t$ we have

$$
\begin{aligned}
& \left|\int_{\Omega} x^{\tilde{N}}(t ; \omega) d P_{k}(\omega)-\int_{\Omega} x(t ; \omega) d P(\omega)\right| \\
& \leq\left|\int_{\Omega}\left(x^{\tilde{N}}(t ; \omega)-x(t ; \omega)\right) d P_{k}(\omega)\right| \\
& +\left|\int_{\Omega} x(t ; \omega) d P_{k}(\omega)-\int_{\Omega} x(t ; \omega) d P(\omega)\right| \\
& \equiv I+I I .
\end{aligned}
$$

For the first term, we find

$$
I \leq \int_{\Omega}\left|x^{\tilde{N}}(t ; \omega)-x(t ; \omega)\right| d P_{k}(\omega) .
$$

Suppose $\epsilon>0$. Choose $N_{0}$ so that $\tilde{N} \geq N_{0}$ implies

$$
\left|x^{\tilde{N}}(t ; \omega)-x(t ; \omega)\right|<\epsilon \quad \text { for all } \omega \in \Omega .
$$

Then for every $k$ we have

$$
\int_{\Omega}\left|x^{\tilde{N}}(t ; \omega)-x(t ; \omega)\right| d P_{k}(\omega)<\epsilon .
$$

Thus $I \rightarrow 0$ as $\tilde{N} \rightarrow \infty$ uniformly in $k$.

Considering the second term, we have

$$
I I=\left[\int_{\Omega} x(t ; \omega) d P_{k}(\omega)-\int_{\Omega} x(t ; \omega) d P(\omega)\right]
$$

But for each $t, x(t ; \cdot)$ is in $C(\Omega)$ and by definition of the Prohorov metric (actually, one of it's equivalent characterizations! [4]), we have immediately that $I I \rightarrow 0$ and the theorem is proved. 
These preliminaries lead to our basic results on convergence (note that condition $\mathrm{C} 2$. below is precisely the results given above in Theorem 3.3):

The following result establishes the computational convergence in the Prohorov Metric Framework for fixed $N$. These results establish a comprehensive body of theory for the least squares estimation of the measure $P_{0}$ that is assumed to have generated the observed data. It is given in [25, Theorem 5.1]:

Theorem 3.4. (Convergence) Let $(\Omega, d)$, with $\Omega=\Omega_{1} \times \Omega_{p}$, be a compact, separable metric space and consider the space $(\mathcal{P}(\Omega), \rho)$ of probability measures on $\Omega$ with the Prohorov metric, as before. Let $\mathcal{P}^{M}(\Omega)$ be as defined as above (e.g., using Dirac or spline approximates for elements of $\Omega$ ). Assume

C1. The map $P \mapsto J_{N}^{\tilde{N}}(v, P)$ is continuous for all $\tilde{N}, N$;

C2. For any sequence of probability measures with $P_{k} \rightarrow P$ in $\mathcal{P}(\Omega), v^{\tilde{N}}\left(t ; P_{k}\right) \rightarrow$ $v(t ; P)$ as $\tilde{N}, k \rightarrow \infty$;

C3. The function $v(t ; P)$ is uniformly bounded for all $t, P$.

Then there exists minimizers $\hat{P}_{N, M}^{\tilde{N}}$ satisfying (4). Moreover, for fixed $N$, there exists a subsequence (as $M, \tilde{N} \rightarrow \infty)$ of the approximate estimates $\hat{P}_{N, M}^{\tilde{N}}$ which converges to some $\hat{P}_{N}^{*}$ which satisfies (2).

This theorem provides a set of conditions under which a sequence of approximate estimates $\hat{P}_{N, M}^{\tilde{N}}$ converges to the estimate $\hat{P}_{N}^{*}$ of interest. This estimate is itself a realization (for a particular data set) of the estimator $P_{N}$ which can be shown to exist and to be consistent (see the next section), so that $P_{N} \rightarrow P_{0}$ with probability one. Thus we are assured that a computed measure $\hat{P}_{N, M}^{\tilde{N}}$ is an accurate estimate of the true distribution $P_{0}$. The conditions assumed in Theorem 3.4 are not restrictive. In typical problems (and, indeed, in the assumptions of other theorems appearing in this document) it is assumed that the parameter space $\Omega$ as well as the independent variable space $T$ are compact. In such a case, Conditions $\mathrm{C} 1$ and $\mathrm{C} 3$ above are satisfied if the individual model solutions $x\left(t ; \tau_{1}, \theta\right)$ are continuous on $T \times \Omega$. Condition $\mathrm{C} 2$ is then simply an assumption on the convergence of the numerical procedures used in obtaining model solutions which we have established above! This result is, in essence, a verification of an analogue for our problems of hypothesis (iv) of Theorem 3.1 of $[17]$. 


\section{Consistency of the Estimator}

We can next establish consistency for estimators in the case of random delay differential equation problems. The assumptions are essentially the same as those in the case of ordinary differential equation or partial differential equation estimators.

(A1) For any fixed $N=N_{t}$, the error random variables $\left\{\mathcal{E}_{j}\right\}_{j=1}^{N}$ are independent and identically distributed, defined on some probability triple $\left(\Theta, \Sigma_{\Theta}, P_{\Theta}\right)$.

(A2) For $\overrightarrow{\mathcal{E}}=\left(\mathcal{E}_{1}, \ldots, \mathcal{E}_{N}\right), E[\overrightarrow{\mathcal{E}}]=0$ and $\operatorname{Cov}[\overrightarrow{\mathcal{E}}]=\sigma^{2} I_{N}$, where $I_{N}$ is the $N \times N$ identity matrix.

(A3) $(\Omega, d)$ is a separable, compact metric space; the space $\mathcal{P}(\Omega)$ is taken with the Prohorov metric $\rho$.

(A4) For all $j, 1 \leq j \leq N_{t},\left(t_{j}\right) \in \tilde{T}$ for some compact space $\tilde{T}$.

(A5) The model function $v \in C(\mathcal{P}(\Omega), C(\tilde{T}))$.

(A6) There exists a measure $\mu$ on $\tilde{T}$ such that for all $g \in C(\tilde{T})$

$$
\frac{1}{N} \sum_{j, i=1} g\left(t_{j}\right) \equiv \int_{\tilde{T}} g(t) d \mu_{N}(t) \rightarrow \int_{\tilde{T}} g(t) d \mu(t)
$$

(A7) The functional $J_{0}(P)=\int_{\tilde{T}}\left(v\left(t ; P_{0}\right)-v(t ; P)\right)^{2} d \mu(t)$ is uniquely minimized at $P_{0} \in \mathcal{P}(\Omega)$.

Under the assumptions one can prove consistency.

Theorem 4.1. Under assumptions (A1)-(A7), there exists a set $A \in \Sigma_{\Theta}$ with $P_{\Theta}(A)=1$ such that for all $\theta \in A$,

$$
\frac{1}{N} J_{N}(v ; P)(\theta) \rightarrow J_{0}(P)
$$

as $N \rightarrow \infty$ and for each $P \in \mathcal{P}(\Omega)$. The convergence is uniform on $\mathcal{P}(\Omega)$ and moreover, the estimators $P_{N} \stackrel{w^{*}}{\longrightarrow} P_{0}$ as $N \rightarrow \infty$ with probability 1. That is,

$$
P_{\Theta}\left(\left\{\theta \mid P_{N}(v)(\theta) \rightarrow P_{0}\right\}\right)=1 .
$$

Complete proofs of these results are given in [25].

Theorem 4.1 establishes the consistency of the estimator (2). Given a set of data $v$, it follows that the estimate $\hat{P}_{N}$ corresponding to the estimator $P_{N}$ will converge to the true distribution $P_{0}$ under the stated assumptions. We remark that these assumptions are not overly restrictive (compare [17, 27, 28]) though some of the assumptions may be difficult to verify in practice. Assumptions (A3)-(A5) are mathematical in nature and may be verified directly for each specific problem. Assumptions (A1) and (A2) describe the error process which 
is assumed to generate the collected data. While it may be possible to ascertain a priori that the error process satisfies these assumptions (see [13]), one may also use posterior analysis such as residual plots $[27$, Ch. 3] to investigate the appropriateness of the assumptions of the statistical model. Assumption (A6) reflects the manner in which data is sampled and, together with Assumption (A7), constitutes an identifiability condition for the model. The limiting sampling distribution function $\mu$ may be known if the experimenter has complete control over the values $t_{j}$ of the independent variables (e.g., if the $t_{j}$ are measurement times) but this may not always be the case.

\section{Concluding Remarks}

Inter-individual or intra-individual heterogeneity is often ignored in mathematical models. In the above discussions we model heterogeneity using random delay differential equation models. That is, we formulate delay differential equations in which some parameters are random variables. In particular, we are concerned with the ability to recover the parameter distributions without making any a priori assumptions about the probability distributions.

We have illustrated and validated methods where the inverse problems are of the aggregate data/individual model type. We have established existence of estimators in classes of probability distributions, convergence of approximations and consistency of the estimators.

\section{Acknowledgement}

This research was supported in part by the Air Force Office of Scientific Research (HTB) under grant number AFOSR FA9550-15-1-0298.

\section{References}

[1] R. A. Albanese, H. T. Banks, M. V. Evans, and L. K. Potter, Physiologically based pharmacokinetic models for the transport of trichloroethylene in adipose tissue, Bulletin of Mathematical Biology, 64 (2002), 97-131.

[2] H. T. Banks, Approximation of nonlinear functional differential equation control systems, J. Optimiz. Theory Appl., 29 (1979), 383-408.

[3] H. T. Banks, Identification of nonlinear delay systems using spline methods, in: V. Lakshmikantham (Ed.), Nonlinear Phenomena in Mathematical Sciences, Academic Press, New York, NY, 1982, 47-55.

[4] H. T. Banks, A Functional Analysis Framework for Modeling, Estimation and Control in Science and Engineering, CRC Press, 2012. 
[5] H.T. Banks, J.E. Banks, Riccardo Bommarco, A.N. Laubmeier, N.J. Myers, Maj Rundlof, and Kristen Tillman, Analysis of nonlinear delay systems with applications in bumblebee population models, CRSC-TR17-12, Center for Research in Scientific Computation, N. C. State University, Raleigh, NC, June, 2017; Communication in Applied Analysis, 21 (2017), 449-476.

[6] H.T. Banks and D.M. Bortz, A parameter sensitivity methodology in the context of HIV delay equation models, CRSC-TR02-24, August, 2002; J. Math. Biol., 50 (2005), 607-625.

[7] H.T. Banks and J.A. Burns, An abstract framework for approximate solutions to optimal control problems governed by hereditary systems, International Conference on Differential Equations (H. Antosiewicz, Ed.), Academic Press (1975), 10-25.

[8] H.T. Banks and J.A. Burns, Hereditary control problems: numerical methods based on averaging approximations, SIAM J. Control \& Opt., 16 (1978), 169-208.

[9] H. T. Banks, V. Bokil, S. Hu, A. K. Dhar, R. Bullis, C. Browdy, and F. Allmutt, Modeling shrimp biomass and viral infection for production of biological countermeasures, Mathematical Biosciences and Engineering, 3 (2006), no. 4, 635-660.

[10] H. T. Banks, D. Bortz, and S. Holte, Incorporation of variability into the modeling of viral delays in HIV infection dynamics, Mathematical Biosciences, 183 (2003), no. 1, 63-91.

[11] H. T. Banks, D. Bortz, G. Pinter, and L. Potter, Modeling and imaging techniques with potential for application in bioterrorism, Bioterrorism: Mathematical Modeling Applications in Homeland Security, SIAM, 2003, $129-154$.

[12] H. T. Banks, L. W. Botsford, F. Kappel, and C. Wang, Modeling and estimation in size structured population models, LCDS-CCS Report 87-13, Brown University; Proc. 2nd Course on Mathematical Ecology, (Trieste, December 8-12, 1986) (1988), 521-541.

[13] H. T. Banks, J. Catenacci and S. Hu, Use of difference-based methods to explore statistical and mathematical model discrepancy in inverse problems, CRSC-TR15-05, May, 2015; J. Inverse Ill-Posed Problems, 24 (2016), $413-433$.

[14] H. T. Banks and J. L. Davis, Quantifying uncertainty in the estimation of probability distributions with confidence bands, CRSC-TR07-21, December, 2007; Mathematical Biosciences and Engineering, 5 (2008), 647-667.

[15] H. T. Banks and J. L. Davis, A comparison of approximation methods for the estimation of probability distributions on parameters, Applied Numerical Mathematics, 57 (2007), no. 5-7, 753-777. 
[16] H. T. Banks, J. L. Davis, S. L. Ernstberger, S. Hu, E. Artimovich, and A. K. Dhar, Experimental design and estimation of growth rate distributions in size-structured shrimp populations, Inverse Problems, 25 (2009), no. 9, 095003.

[17] H. T. Banks and B. G. Fitzpatrick, Estimation of growth rate distributions in size structured population models, Quarterly of Applied Mathematics, 49 (1991), no. 2, 215-235.

[18] H. T. Banks, K. B. Flores, C. R. Langlois, T. R. Serio, and S. S. Sindi, Estimating the rate of prion aggregate amplification in yeast with a generation and structured population model, Inverse Problems in Science and Engineering, 26 (2018), 257-279.

[19] H.T. Banks and Michele L. Joyner, AIC under the framework of least squares estimation, CRSC-TR17-09, Center for Research in Scientific Computation, N. C. State University, Raleigh, NC, May, 2017; Applied Math Letters, 74, December (2017), 33-45.

[20] H. T. Banks and F. Kappel, Spline approximations for functional differential equations, J. Differential Equations, 34 (1979), 496-522.

[21] H. T. Banks, Z. R. Kenz, and W. C. Thompson, A review of selected techniques in inverse problem nonparametric probability distribution estimation, CRSC-TR12-13, May 2012; J. Inverse and Ill-Posed Problems, 20 (2012), 429-460.

[22] H. T. Banks and K. Kunisch, Estimation Techniques for Distributed Parameter Systems, Birkhausen, Boston, 1989.

[23] H. T. Banks and L. K. Potter, Probabilistic methods for addressing uncertainty and variability in biological models: Application to a toxicokinetic model, CRSC-TR02-27, September, 2002; Mathematical Biosciences, 192 (2004), 193-22.

[24] H.T. Banks, Danielle Robbins and Karyn L. Sutton, Theoretical foundations for traditional and generalized sensitivity functions for nonlinear delay differential equations CRSC-TR12-14, N. C. State University, Raleigh, NC, July, 2012; Math. Biosci. Engr., 10 (2013), 1301-1333; http://dx.doi.org/10.3934/mbe.2013.10.1301

[25] H. T. Banks and W. C. Thompson, Existence and consistency of a nonparametric estimator of probability measures in the Prohorov Metric Framework, International Journal of Pure and Applied Mathematics, 103 (2015), no. $4,819-843$.

[26] H. T. Banks and K. L. Bihari, Modelling and estimating uncertainty in parameter estimation, Inverse Problems, 17 (2001), no. 1, 95-111. 
[27] H. T. Banks and H. T. Tran, Mathematical and Experimental Modeling of Physical and Biological Processes, CRC Press, 2009.

[28] H. T. Banks, S. Hu, and W. C. Thompson, Modeling and Inverse Problems in the Presence of Uncertainty, CRC Press, 2014.

[29] F. Kappel, An approximation scheme for delay equations, in: V. Lakshmikantham (Ed.), Nonlinear Phenomena in Mathematical Sciences, Academic Press, New York, NY, 1982, 585-595.

[30] L. K. Potter, Physiologically based pharmacokinetic models for the systemic transport of trichloroethylene, Ph.D. thesis, North Carolina State Univeristy, August 2001.

[31] L. B. Sheiner, B. Rosenberg, and K. L. Melmon, Modelling of individual pharmacokinetics for computer-aided drug dosage, Computers and Biomedical Research, 5 (1972), no. 5, 441-459.

[32] J. Simmons, W. Boyes, P. Bushnell, J. Raymer, T. Limsakun, A. McDonald, Y. Sey, and M. Evans, A physiologically based pharmacokinetic model for trichloroethylene in the male long-evans rat, Toxicological Sciences, 69 (2002), no. 1, 3-15. 